\title{
La economía política del alivio de la pobreza: el caso de Progresa en México ${ }^{1}$
}

DOI: $10.32870 /$ mycp.v11i31.300

Yuriko Takahashi ${ }^{2}$

Resumen

El presente estudio analiza la distribución del Programa de Educación, Salud y Nutrición (Progresa) de México, en el año 2000 y a nivel municipal, para demostrar que el neoliberalismo no parece eliminar la manipulación política del gasto social, a pesar de las predicciones optimistas hechas por sus defensores. Los resultados muestran que el supuesto fundamental de que la competencia entre dos partidos es la base de los modelos teóricos de políticas distributivas no es sostenible en muchas nuevas democracias porque, generalmente, en la competencia participan más de dos partidos. Destacando las presiones competitivas distintivas que se derivan de las diversas configuraciones partidistas a nivel subnacional, un análisis binomial negativo de la regresión proporciona los siguientes resultados: cuando el gobernante Partido Revolucionario Institucional (PRI) hace frente a una competencia entre tres partidos, en la cual el grado de incertidumbre es mayor que en una competencia entre dos partidos, asigna recursos de manera más favorable a los municipios: a) donde es más fuerte el apoyo político al PRI que a aquellos en los que la carrera es más cerrada, y b) a los municipios que son gobernados por alcaldes del PRI. Este estudio concluye que puesto que la manipulación política del gasto social es un tema importante para el análisis político - aunque, a la vez, sea difícil de confirmar-, la evidencia cuantitativa se debe complementar mediante cuidadosos estudios de caso.

Palabras clave: pobreza, política social, elección, partidos políticos, economía política

1. Quisiera agradecer a Kenneth Roberts, Walter Mebane, Jonas Pontusson, y Christopher Way por sus útiles comentarios, así como a Melba E. Falck por su generosa ayuda. Una versión anterior de este trabajo fue publicada en Journal of International Cooperation Studies, vol. 14, núm. 3, en marzo de 2007. Agradezco a la GISICS, Universidad de Kobe, por permitir la reim-presión de mi trabajo.

2. Profesora de la Universidad de Kobe, Japón. ORCID http://orcid.org/0000-0003-4455-9327 


\begin{abstract}
The present study analyzes the distribution of the Program for Education, Health and Nutrition of Mexico (Progresa) in the year 2000 at the municipal level in order to show that neoliberalism do not seem to eliminate the political manipulation of social expenditure notwithstanding the positive offers by its defenders. The results show that the fundamental assumption that the competition between two political parties is the base of the theoretical models of distributive policies is not sustainable in many democracies because generally in the political competition more than two parties participate. By emphasizing the distinctive nature of competition from different party configurations at the sub national level, a negative binomial analysis of the regression provides the following results: When the governing Institutional Revolutionary Party (PRI) confronts a three party competition, in which the degree of uncertainty is greater than a two party competition, the dominant party distribute resources more favorably to municipalities a) where the support is stronger to PRI than to those in which the competition is more closed and b) to municipalities that are governed by PRI majors. This study concludes that since political manipulation is an important topic for political analysis, but at the same time, difficult to confirm, the quantitative evidence should be supported by case studies.

Key words: Poverty, social policy, elections, political parties, political economy.
\end{abstract}

\title{
Introducción
}

$\mathrm{E}$ 1 alivio de la pobreza es un problema político que tiene alta prioridad para los gobiernos en los países menos desarrollados, porque la expansión de la pobreza ha incluido a gran parte de la población. A pesar de tal imperativo, los gobiernos han hecho frente al dilema de erradicar la pobreza dentro de las estrictas limitaciones presupuestarias impuestas por las reformas económicas neoliberales, que se han expandido rápidamente a muchos países en vías de desarrollo desde los años ochenta. El uso de programas para erradicar la pobreza se ha introducido ampliamente como una solución viable para entregar con eficiencia los escasos recursos a los más pobres, empleando métodos tecnócratas para seleccionar los beneficiarios elegibles (Laurell, 2003: 343). Con respecto a la distribución de los nuevos programas sociales orientados, ha habido un gran debate académico con relación a si son susceptibles a la manipulación política, particularmente en los países menos desarrollados, donde tradicionalmente ha prevalecido la política conducida por el patrocinio. El trabajo anterior sugirió que el neoliberalismo deja poco espacio a la manipulación política de los recursos públicos porque la severidad fiscal, la privatización y la desregulación limitan seriamente las oportunidades políticas y los recursos financieros para la distribución del patrocinio (Heredia, 
1997: 26). En contraste, existen evidencias de que continúa la manipulación política de las asignaciones para gastos sociales. ${ }^{3}$ Estudios recientes muestran que los programas orientados de alivio de la pobreza son asignados según las motivaciones electorales (Bruhn 1996; Graham 1992; Magaloni, Díaz-Cayeros y Estévez, 2003; Molinar y Weldon, 1994; Rocha Menocal, 2001; Schady, 2000). ${ }^{4}$ Teniendo en cuenta esas evaluaciones contradictorias, quedan las siguientes preguntas. ¿Cuáles son las condiciones bajo las que se asignan correctamente los programas orientados? ¿Qué factores están conduciendo a la (des)politización de estos programas? ¿Qué interesa más, el neoliberalismo o la competencia política?

Para abordar estas cuestiones se requiere un análisis longitudinal en el que se comparen los resultados del programa a través de los años y se observe un cambio en las prácticas de gastos con el tiempo. Sin embargo, por razones de espacio, el presente estudio se centra en un programa para combatir la pobreza que

Existen evidencias de que continúa la manipulación política de las asignaciones para gastos sociales fuera introducido en el neoliberalismo, y pretende realizar un análisis en dos etapas para examinar los determinantes de la asignación de ese programa. ${ }^{5}$ Primero, examino si los gobiernos que han introducido reformas económicas neoliberales han eliminado realmente la programación social politizada en favor de una elaboración de políticas más tecnócratas, como dicen oficialmente que hacen. Luego, con un análisis altamente desagregado, examino cómo ocurre la politización "neoliberal", si la hay. Especialmente para esta segunda etapa

3. Barbara Geddes ofrece una explicación general de cómo las limitaciones políticas impiden la aplicación de reformas ampliamente apoyadas en los países menos desarrollados (Geddes, 1994).

4. Un uso político de ese tipo de los programas orientados en el neoliberalismo se analiza en asociación con el aumento del "neopopulismo", que se distingue del populismo "clásico", característico de la política de principios del siglo xx en América Latina. Estudios recientes identifican esta coexistencia intrínsecamente paradójica del populismo y el neoliberalismo como una "amalgama inesperada" (Roberts, 1995) o "afinidades inesperadas" (Weyland, 1996). Sin embargo, este importante argumento está fuera del alcance de este estudio.

5. Mi otro proyecto tiene como objetivo comparar tres programas en México - Pronasol, Progresa y Oportunidades - y explorar las condiciones económicas y políticas que conducen a la despolitización de los programas orientados contra la pobreza. 
del análisis, argumento que asumir que la competencia entre dos partidos es la base de los modelos teóricos existentes resulta insostenible para muchos casos de los países menos desarrollados, porque los sistemas partidistas no se consolidan, y generalmente son más de dos los partidos en competencia. Modificando esta suposición, propongo un modelo econométrico para estimar las estrategias distintivas de la asignación geográfica de los gastos que son contingentes en la característica de la competencia partidista. Para este propósito, me centro en el caso del Programa de Educación, Salud, y Nutrición (Progresa), que fue promovido en 1997 en México por iniciativa del presidente Ernesto Zedillo (1994-2000). Específicamente, empleo datos sobre los resultados electorales, la distribución de gastos de Progresa y los atributos socioeconómicos a nivel municipal. Al emplear datos del conteo de familias beneficiarias de Progresa como medida de los niveles de distribución, realizo una regresión binomial negativa para estimar el efecto de covariables en la distribución de Progresa. Progresa constituye un caso ideal para responder las preguntas de este análisis por dos razones. Primero, Progresa es el caso "menos probable" para examinar la posibilidad de maniobra electoral de los recursos. El presidente Zedillo emprendió una iniciativa para consolidar la reestructuración económica neoliberal e introdujo un criterio estrictamente tecnocrático en el establecimiento de la política. En 1997, Zedillo promovió Progresa en un esfuerzo explícito por disminuir la politización del programa contra la pobreza, con vistas a alcanzar completamente su objetivo declarado de aliviar la pobreza (Escobar Latapí, 2002: 220; Pardinas, 2004: 67). Más específicamente, Zedillo introdujo una medida estrictamente en función de las necesidades económicas, basada en un índice de marginación para seleccionar los beneficiarios del programa. Se suponía que el esfuerzo explícito de Zedillo eliminaría cualquier posibilidad de intervención política en el proceso de selección de los receptores del programa. De esta forma, cualquier evidencia de ello corroboraría la persistencia del uso político del gasto social en los políticos mexicanos, a pesar de las limitaciones neoliberales. En segundo lugar, durante el periodo de Progresa, el número de partidos en competencia variaba, de un municipio a otro, entre uno y cuatro. Esto permite el análisis abarcador de cómo diversas presiones competitivas conducirían a estrategias correspondientemente distintivas del gasto en el caso de dos, tres, o cuatro partidos en competencia.

El estudio se organiza como sigue. En la sección siguiente, describo brevemente el contexto económico político en el cual se diseñó Progresa y 
después examino si las políticas todavía importan en la asignación del gasto con estadísticas descriptivas. En la tercera sección, con el fin de explorar la segunda pregunta en cuanto a qué importancia tiene la política, examino teorías de políticas distributivas y elaboro la hipótesis comprobable. En la cuarta sección, estimo los determinantes de la distribución de los gastos de Progresa en el año de la elección federal de 2000. La sección final concluye con una agenda de investigación futura.

\section{Neoliberalismo, competencia electoral y Progresa en México}

Las tres administraciones anteriores a la actual en México han implementado tres programas contra la pobreza: el Programa Nacional de Solidaridad (Pronasol) durante el gobierno del presidente Carlos Salinas (1998-1994), Progresa del presidente Zedillo (1994-2000), y Oportunidades del presidente Vicente Fox (2000-2006). ${ }^{6}$ La evolución de estos programas ha reflejado el cambio estructural e institucional en la economía y política mexicanas. Como mencionamos anteriormente, la reestructuración económica neoliberal, que se inició en los años ochenta, afectó negativamente a los estratos más bajos de la sociedad y los expuso a mayor riesgo económico y social. El presidente Salinas tomó la iniciativa de introducir Pronasol, que se trataba de un programa orientado, basado en la demanda, para compensarlos por las estrictas limitaciones presupuestarias. Más que beneficiar al más necesitado, Pronasol fue criticado fuertemente por su uso político. En el contexto de la democratización, el Partido Revolucionario Institucional (PRI) necesitaba recuperar el apoyo popular para sobrevivir a la creciente competencia electoral, distribuyendo los beneficios del programa a favor de los distritos electorales seleccionados (Dresser, 1991). ${ }^{7}$ Después de que Salinas fuera desacreditado debido a la crisis del peso y a la inestabilidad política en 1994, así como a la

6. Algunos argumentarían que Oportunidades es simplemente una continuación de Progresa y debido a eso debería considerarse el mismo programa. Aunque los diseños y procedimientos institucionales son básicamente iguales, existen diferencias significativas en los esfuerzos de los políticos para hacer el programa transparente y aplicable reflejando las circunstancias políticas distintivas (coyuntura política) de cada administración. Por esta razón, este estudio los aborda como programas diferentes. Para ser más exacta, Progresa fue renombrado como Oportunidades en el año 2002 durante la administración de Fox.

7. Ha sido creciente el número de estudios que han analizado la motivación política detrás de la distribución de Pronasol (Bruhn, 1996; Hiskey, 2003; Magaloni et al., 2003; Molinar y Weldan, 1994). 
sublevación zapatista y el asesinato de un candidato presidencial, el PRI tuvo que hacer frente a una crisis de legitimidad. Esto indujo a un grupo reformista dentro del partido a hacer que la política pública fuera transparente, eficiente y protegida de los intereses políticos (Scott, 2003: 15-16). En un clima político tal, el presidente Zedillo y sus aliados cercanos hacen un esfuerzo explícito por diseñar un nuevo programa que disminuya la politización y maximice así su objetivo declarado de aliviar la pobreza (Escobar Latapí, 2002: 220; Pardinas, 2004: 67). ${ }^{8}$

A diferencia de Pronasol, Progresa fue un programa de transferencia de efectivo condicional (el llamado CCT), que intentó "romper el círculo vicioso de la pobreza" invirtiendo para crear las capacidades básicas de aquellos con extrema pobreza (Levy, 2006: 21; Trejo y Jones, 1998: 90). La integración de la educación, la salud y la nutrición, así como la estimulación de la responsabilidad compartida y la participación activa son las características fundamentales de Progresa (Trejo y Jones, 1998: 21-22). Más específicamente, las madres son responsables de que sus hijos asistan a las escuelas y se sometan a chequeo médico mensualmente. Para facilitar la responsabilidad compartida, cada comunidad seleccionó a una promotora entre las madres que reciben los beneficios, quien supuestamente ayudaba a divulgar la información del programa y a promover la participación activa en el programa (Poder Ejecutivo Federal, s/f: 57). Al iniciarse el programa en 1997, abarcó a 300,000 familias en 6,344 localidades de doce estados, con un presupuesto de 58.8 millones de dólares norteamericanos. Al final de la administración de Zedillo, el programa se amplió abarcando hasta 2'476,400 familias en 53,232 localidades en todas las entidades federales excepto en el Distrito Federal (Levy, 2006: 26, 31).

Los esfuerzos por despolitizar Progresa se materializaron en el método de selección de los beneficiarios y la introducción de mecanismos de supervisión. Por un lado, se introdujo una estricta verificación de los recursos para la selección de beneficiarios elegibles, con el fin de eliminar una discreción política en la asignación del gasto. Más específicamente, Progresa primero

8. El énfasis de Progresa orientado hacia los que estaban en la extrema pobreza fue propuesto originalmente por el influyente trabajo de Santiago Levy (Levy, 1991). Él y José Gómez de León, un demógrafo, fueron considerados los principales arquitectos de Progresa, y Gómez de León fungió como primer coordinador nacional de Progresa. Estas iniciativas tecnócratas enfrentaron una oposición severa de muchas fuerzas (PAN, PRD, y fracciones del PRI), que retrasaron la promoción del programa hasta agosto de 1997 (IDB, 2004). Ciertamente, debería hacerse un análisis detallado de este proceso político. 
identificaba a las comunidades pobres empleando un índice de marginación desarrollado a partir de los datos del censo. ${ }^{9}$ El índice de marginación se dividía en cinco categorías basadas en el nivel de marginalidad, como muy alto, alto, medio, bajo, y muy bajo, y luego se iniciaba la incorporación al programa por las localidades de nivel muy alto. En segundo lugar, eran elegidas las familias beneficiarias dentro de aquellas comunidades seleccionadas, sobre la base de una encuesta a la familia que examinaba su estatus socioeconómico y su elegibilidad para la ayuda. En tercer lugar, la asamblea dentro de esas comunidades tomaba la decisión final de determinar si las familias seleccionadas como beneficiarias eran realmente elegibles o no (Skoufias, Davis y De la Vega, 2001: 1769, 1771). ${ }^{10}$ La información de los beneficiarios seleccionados a través de este proceso de tres etapas era enviada directamente a la oficina central de Progresa. En el papel, este mecanismo centralizado podría eliminar el "espacio institucional para la mediación entre el gobierno y los agentes sociales organizados", lo que es un incentivo importante para la politización de la asignación de recursos (Laurell, 2003: 343). De esta forma, se esperaba que Progresa no dejara lugar para que el cálculo político interviniese en el proceso de selección de los receptores del programa.

Por otra parte, la supervisión de Progresa se ha establecido y consolidado a partir de 1998. Desde 1998, el decreto del presupuesto federal ha contenido disposiciones con respecto a la transparencia, el acceso a la información, las evaluaciones externas, y los mecanismos de auditoría. Para la evaluación del programa, el Instituto Internacional de Investigación de Alimentos (IFPRI) realizó un experimento para examinar si la implementación del programa tenía impactos distintivos al comparar grupos de control y grupos de tratamiento. ${ }^{11}$ Los antropólogos de CIESAS (Centro de Investigaciones y Estudios

9. El Consejo Nacional de Población (Conapo) creó un índice de marginación para localidades de México, empleando siete variables. Entre ellas se incluyen: 1. La cantidad de población analfabeta con 15 años o más, 2. La cantidad de viviendas sin agua corriente, 3. La cantidad de viviendas sin drenaje, 4. La cantidad de viviendas sin electricidad, 5. El número promedio de inquilinos por habitación, 6. La cantidad de viviendas con piso de tierra, y 7. El porcentaje de la fuerza laboral que trabaja en el sector agrícola (Skoufias, Davis y De la Vega, 2001: 1771; Conapo, 1995).

10. A este nivel de selección del beneficiario, la influencia del líder de la comunidad pudo haber causado una selección discrecional. Sin embargo, John Scott observa que, en la práctica, esta preocupación no era relevante porque las decisiones de la comunidad no tenían impactos reales en la modificación del listado de beneficiarios (Scott, 2003: 7).

11. Los informes están disponibles en el sitio Web de IFPRI: http://www.ifpri.org/themes/progresa.htm. 
Superiores en Antropología Social) publicaron informes sobre la evaluación cualitativa. Además, el decreto estipula que la rama ejecutiva debe emitir reglas de operación claras (reglas de operación), lo que ha contribuido a reducir la selección discrecional de los beneficiarios (Levy, 2006: 103). Santiago Levy señala que el cumplimiento de las reglas operacionales también estaba sujeto a una supervisión por parte del Ministerio de Verificación Fiscal y la Oficina de Auditoría del Congreso (Levy, 2006: 103). ${ }^{12}$

Aunque se introdujo una serie de estos mecanismos de "autocontrol" al menos en teoría, existen algunas razones para suponer que Progresa continua siendo sensible a la manipulación política. La competencia electoral se hizo más intensa durante el periodo de Progresa, y el PRI perdió el estatus mayoritario en las elecciones intermedias de 1997. Esta transición de un sistema hegemónico a uno multipartidista proporcionaría al candidato del PRI un mayor incentivo para manipular el gasto de Progresa, con el fin de sobrevivir en la carrera electoral por las siguientes razones: primero, la fórmula orientada, que distribuye recursos a individuos específicos, podía servir como una estrategia más viable de movilización del voto en la era del neoliberalismo, que ha debilitado a los intereses sociales organizados tales como los sindicatos. Particularmente, la naturaleza directa, selectiva y visible de la asignación orientada podría crear más vínculos de clientelismo fuerte que beneficios universales, y de esta forma fortalecer los incentivos para el intercambio político (Roberts, 1995: 91).

En segundo lugar, dado el elevado número de pobres, una amplia cobertura del programa contra la pobreza es atractiva políticamente porque es más posible que los pobres sean susceptibles a la compra del voto. Como demuestran los análisis formales, mientras más ricos se hagan, menor será la utilidad marginal que surge de los beneficios particularistas (Brusco, Nazareno y Stokes, 2004; Calvo y Murillo, 2004; Magaloni, Díaz-Cayeros y Estévez, 2003; Robinson y Verdier, 2002; Medina y Stokes, 2002; Stokes, 2005). La cobertura de Progresa comprende hasta 2,000 de las 2,438 municipalidades de México en $1999,{ }^{13}$ lo que implica un gran potencial para comprar el apoyo de esos beneficiarios pobres.

12. Además de estos mecanismos para ampliar la responsabilidad horizontal, los actores de la sociedad civil aumentaron su influencia y ejercieron significativas presiones para hacer los programas sociales más transparentes y confiables durante ese periodo.

13. Estas estadísticas básicas de Progresa aparecieron en el informe de IFPRI. 
En tercer lugar, los resultados de las reformas económicas neoliberales no eran iguales para todo el país, permitiendo la posibilidad de que la selección discrecional de los beneficiarios del programa continuara aun a nivel subnacional (Fox, 1994; Snyder, 2001). Particularmente, la influencia de los gobiernos municipales constituía una cuestión problemática. Al realizar la encuesta a la familia para determinar los beneficiarios del programa, los trabajadores sociales tenían que contar con la suficiente información sobre las localidades que debían visitar. Sin embargo, a menudo carecían de mucha información local, y, por tanto, tenían que apoyarse en la ayuda de los gobiernos locales. Hubo casos en que visitaron con más frecuencia aquellas localidades propuestas por los gobiernos municipales, que tenían un potencial de influencia en la selección de los beneficiarios (Adato, Coady y Ruel, 2000: 33). Esta clase de limitación técnica hizo el proceso de selección menos transparente, y pudo haber dado lugar a la intervención política de los agentes locales (funcionarios, promotores, profesores y doctores municipales) en algunas regiones (Scott, 2003: 39-40). ${ }^{14}$

De hecho, durante las elecciones federales de 2000 se presentó evidencia significativa de manipulación de Progresa con fines electorales a nivel local (Alianza Cívica, 2000; Mariscal, 2000). ${ }^{15}$ Por ejemplo, algunos miembros del partido, sobre todo del PRI, amenazaron a los beneficiarios con que les retirarían los beneficios si no votaban por el candidato del PRI. A pesar de estas múltiples posibilidades y de la evidencia de manipulación política, ninguna de las evaluaciones externas de Progresa tomó en cuenta la dimensión política (Scott, 2003: 39). Para verificar si la asignación de Progresa sigue o difiere de los criterios de pobreza, las figuras 1 y 2 muestran la relación entre la distribución geográfica de Progresa y el índice de pobreza a nivel estatal y municipal. Si la distribución de Progresa difiere del objetivo original decla-

14. Esta posible influencia de los actores municipales también fue confirmada por un funcionario de alto nivel de Sedesol (entrevistado el 23 de febrero del 2006, en la Ciudad de México). Los trabajadores sociales de Veracruz platearon que los gobiernos municipales intentan participar activamente en la implementación del programa durante el periodo de Progresa. Pero esta tentativa disminuyó después que se fortaleciera el cumplimiento de las reglas de operación para Oportunidades (entrevistado el 18 de septiembre de 2006).

15. Flacso (Facultad Latinoamericana de Ciencias Sociales) fue seleccionada por el Instituto Electoral Federal (IFE) para realizar una investigación nacional sobre la compra de votos para las elecciones federales de 2000 (Del Pozo y Aparicio, 2001). Encontraron evidencia de que los programas sociales fueron utilizados para la compra de votos, pero no especificaron qué programas sociales fueron manipulados. 
rado de alivio de la pobreza, ésta sería una fuerte evidencia de la motivación política continuada detrás del programa social del gobierno. La figura 1 presenta la distribución geográfica de los fondos de Progresa per cápita en el año 2000, calculada por cada estado. Los estados están organizados según el índice de marginación antes mencionado (el más pobre a la izquierda y el más acomodado a la derecha). Puesto que, teóricamente, el programa de alivio de la pobreza se asigna según el nivel de pobreza, se espera que el nivel de distribución sea más alto en los estados más pobres y más bajo en los estados menos pobres. La figura 1 muestra que Progresa se distribuye de modo más favorable a los estados más pobres. Este hallazgo sugiere que la asignación de Progresa obedece más al objetivo del programa orientado a los pobres que a tener su base en el cálculo político.

Figura 1

Distribución de Progresa por Estado

(Transferencia per cápita, 2000)

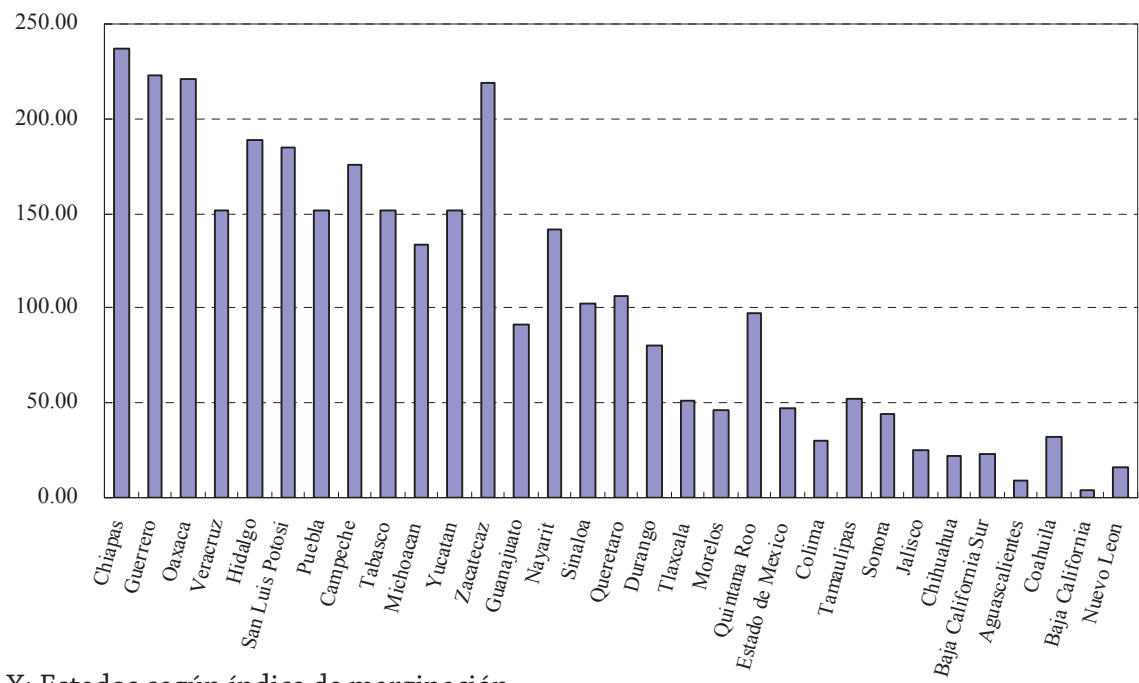

X: Estados según índice de marginación.

Y: Pesos mexicanos.

Fuente: Zedillo Ponce de León. 2000. Sexto Informe del Gobierno.

Sin embargo, dada la composición heterogénea de las poblaciones del estado, es prematuro concluir que Progresa está libre de cálculo político sobre la base de un análisis a nivel del estado. En otras palabras, debido a que la mayoría 
de los estados incluyen municipios ricos y pobres, es difícil saber cómo están distribuidos realmente los fondos con datos agregados a nivel estatal. Por tanto, se requiere un análisis desagregado adicional para mostrar una relación más exacta entre el gasto y la pobreza. La figura 2 muestra la asociación entre la distribución de Progresa en el año 2000 y la pobreza a nivel municipal. Debido a que los datos de los gastos a este nivel no estaban disponibles, la proporción de beneficiarios de Progresa con respecto a la población total del municipio se traza contra el índice de marginación, que oscila entre 1 -el menos pobre- - 5 - el más pobre- La línea de regresión no paramétrica dibujada en el diagrama de dispersión muestra la relación entre el nivel de beneficio de Progresa y la pobreza. ${ }^{16}$

Figura 2

Distribución de Progresa y Pobreza por Municipios ( $\mathrm{N}=2387$ )

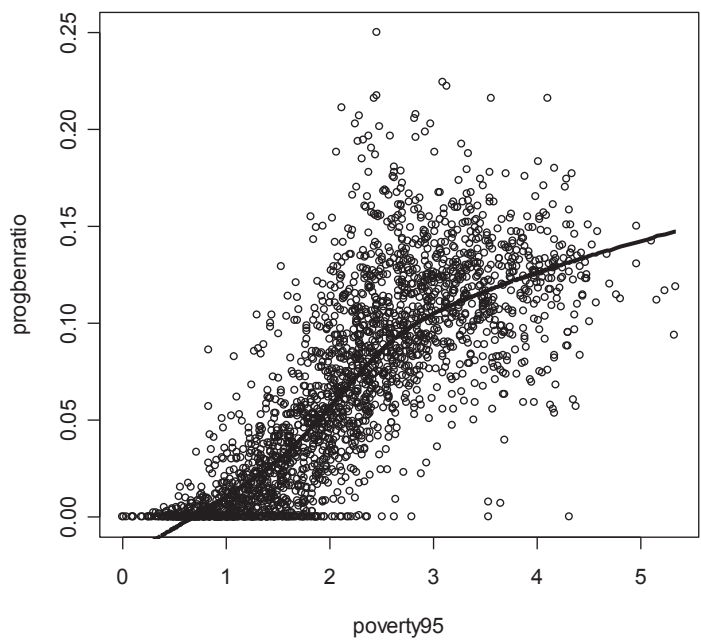

Y: La proporción de los beneficiarios de Progresa con respecto a la población total por municipio (2000)

X: Nivel de pobreza medido por el índice de marginación ( 1 = menos pobre, 5 = más pobre) (1995)

Excluyendo un valor discrepante (Pitiquito, Sonora)

16. Una regresión no paramétrica no impone asunciones sobre formas funcionales. Esta fórmula menos limitada permite presentar una relación más exacta entre las variables que la de los mínimos cuadrados (OLS) (Schady, 2000: 296). 
Recuerde que el índice de marginación fue calculado por métodos de componentes principales, empleando siete variables que representaban la "parte" de la población en condiciones de vida desfavorables específicas (Skoufias, Davis y De La Vega, 2001: 1771; Conapo, 1995). Siguiendo esta fórmula, el municipio más pobre, indexado como 5 , representa un municipio con el porcentaje más alto de la población pobre. Si Progresa se orienta a los pobres y pone la prioridad más alta en la pobreza extrema, la curva debe ser ascendente, y la proporción de los beneficiarios de Progresa con respecto a la población municipal total supuestamente debe aumentar en proporción al nivel de pobreza. En la práctica, sin embargo, la curva no paramétrica sugiere que el nivel de beneficio aumenta por encima del nivel de pobreza medio, pero una vez que exceda ese nivel, la tasa de incremento marginal disminuye, lo que implica un proceso artificial de incorporar las familias beneficiarias por encima del nivel medio de pobreza.

En resumen, sería razonable plantear que, indudablemente, Progresa fue favorable a los pobres al orientar los fondos a favor de dicha población, pero el nivel de beneficio no es directamente proporcional al nivel de pobreza. Estos resultados sugieren una posibilidad de que en el proceso de la selección del beneficiario de Progresa pudieran haber intervenido cálculos políticos. En la medida en que la política importe, ¿qué tipo de motivaciones políticas pueden ser discernidas? La sección siguiente presenta argumentos teóricos para extraer hipótesis comprobables sobre lógicas políticas plausibles detrás de la asignación de los gastos de Progresa.

\section{¿Qué importancia tiene la política? Competencia electoral y Progresa}

Como mencionamos brevemente en la sección anterior, el aumento de la presión electoral, que ha coincidido con el periodo de reestructuración económica neoliberal, pudo motivar a que políticos ambiciosos manipularan persistentemente el gasto social para fines políticos. Hasta la fecha, el trabajo sobre programas contra la pobreza se ha centrado en tres aspectos distintivos de la política electoral para determinar el efecto de la competencia electoral en la asignación de los gastos sociales: modelos geográficos de votación, condiciones específicas de la competencia partidista, y partidismo municipal. El debate en el trabajo previo giró en gran parte alrededor del primer enfoque. El problema inherente en este enfoque es que asume implícitamente la competencia entre 
dos partidos, lo cual no es válido para las nuevas democracias en las cuales, por lo general, hay más de dos partidos en competencia. Para una valoración exacta de los determinantes de la distribución de Progresa, planteo que el análisis empírico debería probar conjuntamente las propuestas tomadas del primer enfoque con el efecto condicional de variar las configuraciones partidistas, que es el planteamiento central del segundo enfoque.

Antes de proponer mi modelo empírico, analizo las hipótesis competitivas sobre los determinantes de la politización del gasto. Haciendo inferencias de los últimos resultados electorales, el primer enfoque ha debatido la relación entre la asignación geográfica de los patrones del gasto y la votación. Esta línea de investigación es motivada por las predicciones extraídas de modelos teóricos de la política distributiva, particularmente, de la de hipótesis del votante principal y del votante oscilante (Case, 2001; Magaloni, Díaz-Cayeros y Estévez, 2003; Schady, 2001). Si se asume que los partidos son adversarios en riesgo, Cox y McCubbins (1986) afirman que al enfrentar incertidumbre, tienen un gran incentivo para invertir más favorablemente en los partidarios principales porque se supone que traen una mayor tasa de beneficio que la oposición o los votantes oscilantes (hipótesis del votante principal). Por otra parte, los modelos del votante oscilante implican que los partidos gastan más en carreras cerradas debido a que un número pequeño de votantes oscilantes puede ser decisivo en el resultado electoral. Gastar más en carreras cerradas, es más rentable porque los partidos tienen poco incentivo para desviar los escasos recursos a las carreras donde saben con certeza que ganarán o perderán (Lindbeck y Weibull, 1987; Dixit y Londregan, 1996) (hipótesis de la proximidad). Además de ese nivel de apoyo político, a los políticos también les puede interesar un cambio reciente en los patrones de votación. Más específicamente, los candidatos pueden intentar "recomprar" a los partidarios tradicionales que votaron recientemente por los candidatos de la oposición (Bruhn, 1996; Schady, 2000) (hipótesis de la recompra). ${ }^{17}$ Alternativamente, los candidatos también podrían hacer un esfuerzo explícito por consolidar nuevos partidarios que votaron por el partido por primera vez en las elecciones anteriores (hipótesis del cultivo) (ibíd.).

17. Hasta donde conozco, Schady hizo el primer intento de clasificar explícitamente esos patrones de votación en cambio y nivel y de incorporar el aspecto dinámico a un modelo empírico (Schady, 2000: 290). 
Los estudios empíricos que promueven dicho enfoque prueban estas hipótesis competitivas, pero sus resultados pueden basarse en valoraciones inexactas porque obedecen implícitamente a la premisa de que la competencia entre dos partidos es la base de los modelos teóricos. Más específicamente, esta premisa, basada en las experiencias de los Estados Unidos, no es sostenible para muchos de los países menos desarrollados porque los sistemas partidistas no están consolidados, y, por lo general, hay más de dos partidos en competencia. En estas variables de configuraciones partidistas, se supone que las estrategias de un candidato para manipular los recursos dependen de si enfrenta a uno, dos, o más adversarios serios, porque a medida que aumenta el número de partidos, es mayor el grado de incertidumbre. ${ }^{18}$ Supongamos que existen tres partidos $P_{1}, P_{2}, P_{3}$ en el distrito $i$. Ahora bien, hay que tener en cuenta que ninguno de ellos constituye un partido trivial, sino que los tres son asumidos como competidores serios. En este caso, el resultado electoral, es decir, si $P_{1}, P_{2}$, o $P_{3}$ ganaran, es menos seguro que en una carrera entre dos partidos. Si cuatro partidos compitieran para un puesto, el resultado sería incluso menos predecible.

La figura 3 proporciona evidencia de que la distribución de Progresa pudo haber variado, dependiendo de la naturaleza de la configuración partidista. La misma desagrega la figura 2 por tipos de competencia partidista medida por el número efectivo de partidos (en adelante ENP). El ENP se define como "el número de partidos hipotéticos de igual tamaño que tendrían el mismo efecto total en el fraccionamiento del sistema que tendrían los partidos reales de tamaño desigual" (Laakso y Taagepera, 1979: 4, énfasis en el original). Lo que interesa aquí no es el número total de partidos, incluyendo los muy pequeños, sino el tamaño relativo de los partidos que constituyen amenazas realmente influyentes y creíbles para el candidato (Laakso y Taagepera, 1979: 3). Empleando el índice de Laakso-Taagepera, clasifico los municipios de México por el número efectivo de partidos que compitieron en la elección de 1997. Los municipios donde el ENP es menor que 1.5 se categorizan como competencia del partido hegemónico; donde el ENP es mayor que 1.5 pero menor que 2.5, como competencia entre dos partidos; donde el ENP es mayor que 2.5 pero

18. Por ejemplo, muchos estudios de los determinantes del Programa Nacional de Solidaridad (Pronasol) en México asumen que las carreras electorales son disputadas entre dos partidos, es decir, PRI contra PAN o PRI contra PRD. De esta forma, no tienen en cuenta los casos en los que los tres partidos importantes compiten cara a cara (Bruhn, 1996; Rocha Menocal, 2000; Molinar y Weldon, 1994). 
menor que 3.5, como competencia entre tres partidos; y donde el ENP es mayor que 3.5, como competencia multipartidista. ${ }^{19}$ Una comparación de las relaciones entre el nivel de pobreza y el nivel de beneficios de Progresa, que se representa en una curva no paramétrica, sugiere que el PRI pudiera haber distribuido beneficios de Progresa de forma diferente, en dependencia de la configuración partidista. ${ }^{20}$

El segundo enfoque muestra que la configuración específica de la competencia partidista distingue las estrategias de distribución (Hiskey, 2003; Magaloni, Díaz-Cayeros y Estévez, 2003). No obstante, el análisis empírico en el trabajo previo necesita más elaboración. Más específicamente, Magaloni, Díaz-Cayeros y Estévez (2003) concluyeron que el Partido Revolucionario Institucional (PRI) de México diversificó las estrategias de inversión de Pronasol, dependiendo de si el municipio estaba caracterizado por ausencia de competencia electoral, un partido hegemónico, o por competencia multipartidista clasificado por el ENP, y que el PRI invirtió más bienes privados en los partidarios principales cuando había menos presiones competitivas. Estos nuevos descubrimientos no son totalmente convincentes porque no se obtuvieron a partir del análisis estadístico sino de una figura que muestra diferentes relaciones entre el nivel de asignación de bienes privados y los efectos del nivel de pobreza al distinguir entre los municipios no competitivos, hegemónicos y competitivos (Magaloni et al., 2003: 29). Para estimar sistemáticamente el efecto de la configuración partidista en las estrategias de distribución, propongo un modelo econométrico para probar las hipótesis competitivas mencionadas con estas presiones competitivas distintivas incorporadas. Considero que este modelo estimará con mayor exactitud la lógica política detrás de la manipulación neoliberal del gasto social. Sobre la base de esta modificación, las hipótesis del principal, de la proximidad, de

19. Magaloni et al. (2003) distinguen entre los municipios sin competencia electoral (el PRI obtuvo $100 \%$ de los votos), municipios hegemónicos donde "había cierta presencia de la oposición, pero el número efectivo de partidos [...] era menor que 1.7", y municipios competitivos donde el número efectivo de partidos es mayor que 1.7. Sin embargo, en las elecciones de 1997, que coincidieron con la fase de implementación de Progresa, no hubo un municipio en el que el PRI recibiera el $100 \%$ de los votos. Por esta razón, la clasificación en este estudio no incluye "ninguna competencia electoral".

20. Aparentemente, cuanto mayor es el número de partidos en competencia, más fuerte es la correlación entre los beneficios y los niveles de pobreza, pero ello debe ser probado estadísticamente, controlando el efecto de otros factores relevantes. 


\section{Figura 3}

Distribución de Progresa y pobreza por la Competencia de Partidos
(a) Partido Hegemónico $(\mathrm{N}=120)$
(b) Competencia entre dos partidos $(\mathrm{N}=1327)$
$(\mathrm{ENP}<1.5)$
$(1.5 \leq \mathrm{ENP}<2.5)$
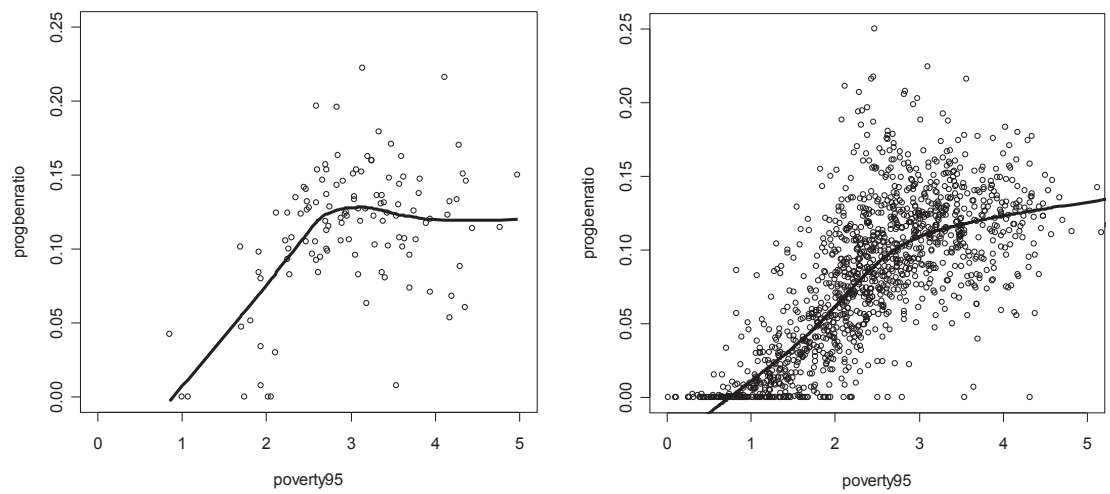

(c) Competencia entre tres partidos $(\mathrm{N}=881)$ $(\mathrm{N}=59)(2.5 \leq \mathrm{ENP}<3.5)$

(d) Competencia entre varios partidos $(\mathrm{ENP} \leq 3.5)$
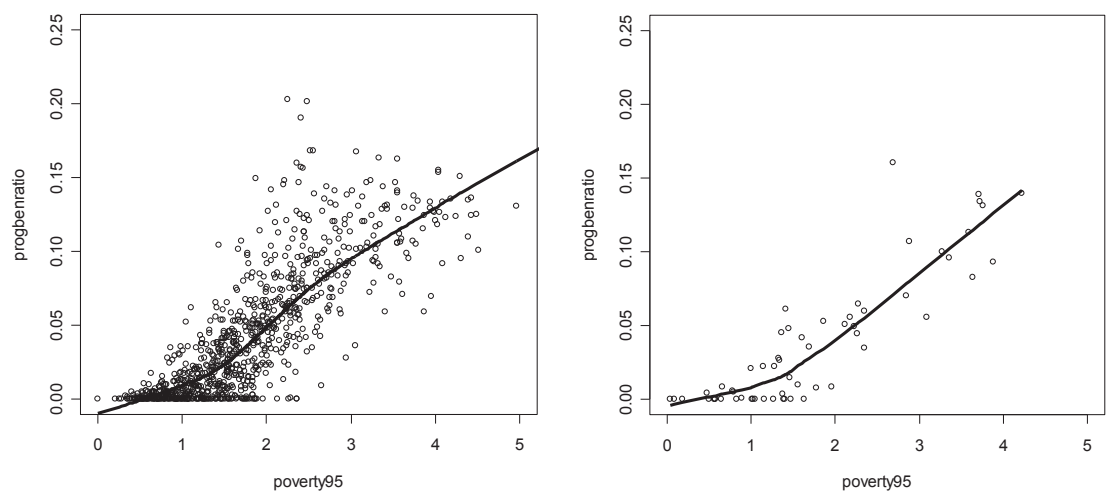
recompra, y de consolidación probadas en este análisis se reformulan de la siguiente manera.

Hipótesis del votante principal. El mayor apoyo al candidato estará asociado a transferencias más grandes. Esta predicción tendría mayor apoyo a medida que aumente el número de contendientes serios, debido a que el candidato tiene más riesgo en contra.

Hipótesis de la proximidad. Una carrera más cerrada atraería más recursos. A medida que aumenta el número de partidos en competencia, también se incrementa el incentivo para invertir en carreras cerradas.

Hipótesis de cultivo. Un mayor incremento de los votos conducirá a mayores transferencias. El candidato se hace más sensible a un cambio en la cantidad de votos cuando el número de competidores en una carrera es mayor.

Hipótesis de recompra. Una mayor disminución del número de votos conducirá a mayores transferencias. El candidato se hace más sensible a un cambio en la cantidad de votos cuando el número de competidores en una carrera es mayor.

Como hemos analizado hasta el momento, el primer y segundo enfoques asumen que el gobierno central puede inferir una opción de voto de un individuo al tener en cuenta los patrones geográficos de las elecciones pasadas y entregar beneficios directamente a votantes específicos. Por el contrario, el tercer enfoque supone que los candidatos ven a los líderes locales como sus clientes partidistas que pudieran servir como corredores para entregar servicios del gobierno central a los beneficiarios y facilitar el intercambio entre votos y beneficios materiales (Calvo y Murillo, 2004; Valenzuela, 1977) ${ }^{21} \mathrm{El}$ gobierno federal necesita manipular con éxito la política meta. Para dirigirse con efectividad a votantes específicos, el gobierno central debe conocer quién apoya a los candidatos o quiénes son votantes marginales, pero es poco plausible supervisar la preferencia de partido de una gran cantidad de individuos. El control del líder local sobre la red del partido ayudaría a solucionar este dilema porque el mismo podría identificar a los seguidores y a los opositores del partido y asegurar la victoria electoral incluso en una elección secreta (Stokes, 2005).

Oficialmente, Progresa está diseñado como un programa altamente centralizado. Ahora bien, parece posible que los alcaldes puedan manipular la

21. Además del partidismo, Calvo y Murillo (2004) enfatizan los efectos institucionales en el acceso de un partido a los recursos fiscales, que genera la distribución geográfica de los votos. 
distribución del programa interviniendo en la etapa final de la selección del beneficiario a nivel local, como hemos señalado anteriormente. Por tanto, la filiación partidista de los gobiernos de nivel inferior determinaría la distribución del gasto. Aunque un examen de cómo los alcaldes distribuyen realmente las transferencias dentro de los municipios requiere estudios de caso detallados, se puede predecir, al menos, que los municipios cuyos alcaldes pertenecen al partido del candidato recibirán transferencias más abundantes. Este efecto también debe probarse clasificando las condiciones de los ambientes electorales locales, que representan grados distintivos de incertidumbre. Anticipo que cuando la carrera es más competitiva e incierta, el candidato tendrá un mayor incentivo para centrarse en los clientes partidistas para asegurar el resultado previsto de la manipulación. Por consiguiente, la hipótesis del clientelismo se formula como aparece a continuación.

Hipótesis del clientelismo. Los municipios gobernados por el partido del candidato reciben mayores transferencias. Con un mayor grado de incertidumbre vinculado a las carreras con un mayor número de candidatos, los candidatos tienen un mayor incentivo para manipular la asignación del gasto a través de los alcaldes que sirven como intermediarios.

A fin de probar estas cinco hipótesis, la siguiente sección presenta un análisis empírico para estimar los efectos de la competencia política en la distribución de los beneficios de Progresa en el año 2000 cuando tuvieron lugar las elecciones federales, y, por lo tanto, la motivación para politizar los programas sociales pudo haber sido mayor.

\section{Distribución geográfica de Progresa}

\section{Diseño de la investigación y especificación del modelo}

Para probar las hipótesis antes mencionadas, este estudio utiliza datos de una muestra representativa de los gastos de Progresa en el año 2000. También utiliza datos sobre los resultados electorales y la pobreza a nivel municipal para analizar los efectos de la motivación política detrás de la distribución geográfica de los beneficios de Progresa. Las elecciones federales fueron realizadas en el año 2000 en México. Para examinar la politización de la distribución de los gastos, los datos del gasto para el año electoral son ideales para demostrar cómo los políticos trataron de politizar los programas sociales durante la campaña electoral previa a la elección. La unidad de análisis es el municipio porque 
no existen datos individuales sobre el comportamiento de la votación de los beneficiarios de Progresa. Al emplear los datos sobre los patrones geográficos de votación a nivel municipal, se aproximan las preferencias de los votantes. Este análisis incluye observaciones para 2,383 del total de 2,436 municipios para los cuales estaban disponibles los datos. ${ }^{22}$ El conjunto de datos no incluye a la Ciudad de México porque antes del año 2000 no se asignaron gastos de Progresa a la capital. Sin embargo, este conjunto de datos incluye un mayor número de observaciones que el análisis a nivel de estado empleado en estudios anteriores, por lo que constituye una muestra más representativa dentro del país. ${ }^{23}$ Ello permite una valoración más exacta de los efectos políticos en los gastos de Progresa. Los datos sobre la distribución geográfica de los gastos a nivel municipal son ideales para determinar el efecto de la competencia partidista en la asignación de Progresa. Sin embargo, no se dispone de los datos del gasto desagregados en una unidad tan pequeña. Alternativamente, el número de familias que reciben Progresa por municipios está disponible, y lo utilizo para este análisis. Puesto que la variable dependiente es un conteo del número de familias beneficiarias que son números enteros no negativos, este análisis desarrolla un modelo empírico de la regresión de los datos del conteo para analizar la relación entre la competencia partidista y el nivel de asignación de Progresa. La distribución de Poisson se utiliza comúnmente para la regresión de los datos del conteo asumiendo la igualdad de la variación condicional y la media, que se define como equidispersión. ${ }^{24}$ Sin embargo, es muy probable que los datos empleados en este análisis estén sobredispersados porque los procesos para producir el número de familias beneficiarias de Progresa en cada municipio pueden diferir substancialmente de un municipio a otro: algunos municipios tienen más personas pobres que otros. Más específicamente, los

22. Se debe mencionar que este análisis se centra en cómo el PRI reacciona a la presión competitiva en el contexto de su hegemonía declinante. Esto asume que el PRI es el primer candidato en la carrera electoral. De hecho, sin embargo, éste es el caso para sólo 1,898 de los 2,383 municipios. Comparé el modelo restringido a estos casos con el modelo con una muestra completa para ver si utilizaría la regresión solo para los casos en los cuales el PRI es el primer candidato. Esta prueba no produjo ninguna diferencia significativa entre los dos modelos. Para incluir toda la información en el análisis, decidí utilizar la muestra completa para este análisis.

23. Hasta donde conozco, Rocha Menocal realizó el primer intento de analizar los determinantes de la distribución de Progresa (Rocha Menocal, 2001). Sin embargo, los resultados extraídos de su análisis a nivel de estado podrían ser engañosos debido a una alta heterogeneidad de configuraciones socioeconómicas y políticas dentro de cada estado de México.

24. La violación del supuesto de Poisson de la equidispersión es equivalente a la falta de asunción de la homoesquedasticidad en el modelo de la regresión lineal (Cameron y Trivedi, 1998: 77). 
datos están sobredispersados "siempre que las probabilidades de selección varíen entre los individuos en cada unidad de observación, pero los grupos de individuos dentro de cada unidad tienen probabilidades similares" (Mebane y Sekhon, 2004: 394) ${ }^{25}$ Una prueba de sobredispersión dio lugar al rechazo de la hipótesis nula de la equidispersión. ${ }^{26}$ De este modo, en lugar de emplear el modelo de la regresión de Poisson, se empleó un modelo binomial negativo de regresión usando un estimador de máxima probabilidad. ${ }^{27}$

En lo que aparece a continuación, primero estimo un modelo incondicional y luego un modelo condicional en los cuales examino los efectos de variables independientes condicionales en la naturaleza de configuraciones partidistas. El modelo binomial negativo incondicional adquiere la siguiente fórmula:

$$
\mathrm{E}\left(\mathrm{y}_{i} \mid \mathrm{x}_{i}\right)=\mu_{i}=\exp (\mathrm{X} \beta)=\exp \left(\sum_{k=1}^{n} \mathrm{X}_{k} \beta_{k}+\gamma_{j}+\varepsilon_{i}\right),
$$

y la función de variación es, ${ }^{28}$

$$
\mathrm{V}\left(\mathrm{y}_{i} \mid \mathrm{x}_{i}\right)=\omega_{i}=\mu_{i}+\alpha \mu_{i}^{2},
$$

donde $\mathrm{E}\left(\mathrm{y}_{i} \mid \mathrm{x}_{i}\right)$ es el valor previsto de la variable dependiente; $\mathrm{X}$ es un vector de variables independientes; $\beta$ es un vector de parámetros estimados; $i$ y $j$ se refieren a las unidades de muestras representativas; $k$ es el número de variables independientes; $\gamma_{j}$ se refiere a las variables simuladas del estado que controla los efectos específicos de la región para cada uno de los 31 estados de México; y $\varepsilon_{i}$ es un término errado.

En el modelo condicional, las variables independientes interactúan con las variables simuladas para el partido hegemónico, la competencia entre dos partidos, la competencia entre tres partidos, y la competencia entre varios

25. La manera en que tales fenómenos de agrupamiento crean una distribución binomial negativa se explica de forma más técnica en McCullagh y Nelder (1989: 198-199) y Mebane y Sekhon (2004: 394).

26. El modelo de regresión de Poisson con la sobredispersión produce "errores estándares estimados falsamente pequeños", lo que conduce, por consiguiente, a valores falsamente grandes (Cameron y Trivedi, 1986: 31).

27. Estas estimaciones fueron computadas empleando la función de glm.nb de R (R Development Core Team, 2004).

28. El modelo binomial negativo despeja el supuesto restrictivo de equidispersión. Existen dos posibilidades de función de variación: $\omega_{i}=(1+\alpha) \mu_{i}$ y $\omega_{i}=\mu_{i}+\alpha \mu_{i}^{2}$. La prueba de sobredispersión confirma que la variación es cuadrática en la media (Cameron y Trivedi, 1998: 63). Así, la función de variación se define como $\omega_{i}=\mu_{i}+\alpha \mu_{i}^{2}$ en este análisis. 
partidos en una sola ecuación. ${ }^{29}$ Como hemos analizado en la sección anterior, utilizo el índice de Laakso-Taagepera para clasificar los municipios de México por el ENP en estas condiciones distintivas de la competencia partidista. La disposición del modelo de interacción se presenta como sigue:

$$
\begin{aligned}
\mathrm{E}\left(\mathrm{y}_{i} \mid \mathrm{x}_{i}\right) & =\mu_{i}=\exp (\mathrm{X} \beta)=\exp \left(\sum_{k=1}^{n} \mathrm{X}_{k 1} \beta_{k 1} * \text { Hegemónico }+\sum_{k=1}^{n} \mathrm{X}_{k 2} \beta_{k 2} *\right. \text { Bipartidista } \\
& \left.+\sum_{k=1}^{n} \mathrm{X}_{k 3} \beta_{k 3} * \text { Tripartidista }+\sum_{k=1}^{n} \mathrm{X}_{k 4} \beta_{k 4} * \text { Multipartidista }+\gamma_{j}+\varepsilon_{i}\right),
\end{aligned}
$$

donde todas las notaciones variables son iguales a las del modelo incondicional.

Comparando los coeficientes estimados para las mismas variables independientes entre los tipos de competencia partidista, se espera que el PRI tenga gastos diferentes, dependiendo del número de partidos en competencia. A continuación se presenta la descripción de cada variable.

\section{Variable dependiente: el número de familias beneficiarias de Progresa}

El número de familias beneficiarias de Progresa por municipio se calcula como sigue. Los datos sobre los receptores de Progresa en el año 2000 en cerca de 52,000 localidades son agregados por municipio ( $\mathrm{N}=2.436)$. Una de las características distintivas de Progresa es su enfoque integrado. Es decir, intenta erradicar las "causas de origen" de la pobreza extrema centrándose en el desarrollo del capital humano. Para alcanzar esta meta, los beneficios cubren la nutrición, la salud y la educación en conjunto, y estos beneficios combinados van a los beneficiarios. Específicamente, los beneficios efectivos están orientados directamente a las familias como unidades beneficiarias con la condición de que envíen a sus niños a la escuela y visiten regularmente los centros de salud (Skoufias, Davis, y De La Vega 2001: 1769). Como resultado, se supone que los beneficios recibidos por cada familia elegible sean homogéneos, es decir, el número de receptores se interpreta como el nivel de beneficios

29. Según Hanushek y Jackson, si la hipótesis de que las variaciones residuales pueden ser reagrupadas es correcta, esta medición produce estimaciones más eficientes del parámetro que opera regresiones por separado para los subconjuntos de datos porque se basa en la información total de toda la muestra reunida (Hanushek y Jackson, 1977: 128). Por ejemplo, esta clase de modelo de interacción es utilizado por Rueda y Pontusson (2000). 
en los municipios. De esta manera, los datos del conteo pueden servir como medida del nivel de gastos de Progresa que se asigna a cada municipio.

\section{Variables independientes}

Para examinar la politización plausible de los gastos, se debe controlar el nivel de pobreza porque, de acuerdo con el propósito original del programa, se supone que el programa contra la pobreza esté orientado a las regiones más pobres. Para la variable Pobreza empleo un índice de marginación calculado por el Conapo (Consejo Nacional de Población). Este índice de marginación se construye por el método de componentes principales basado en siete variables. Éstas son la cantidad (1) de población analfabeta con 15 años o más, (2) de viviendas sin agua corriente, (3) de viviendas sin drenaje, (4) de viviendas sin electricidad, (5) de viviendas con piso de tierra, (6) el número promedio de inquilinos por habitación, y (7) el porcentaje de mano de obra que trabaja en el sector agrícola (Skoufias, Davis y De La Vega, 2001: 1771; Conapo, 1995). Para controlar otros efectos socioeconómicos, primero incluí las variables de PIB per cápita, urbanización, y la proporción de población indígena a nivel municipal. Sin embargo, todas estas variables están fuertemente correlacionadas con el índice de marginación a un nivel estadístico importante. Por esta razón, decidí eliminarlas y emplear exclusivamente el índice de marginación como medida del nivel de pobreza. El signo positivo del coeficiente significa que los gastos de Progresa están orientados a los pobres. Además, el logaritmo de la población en municipios y un término cuadrático (Povsq) y el polinomio de tercer grado (Pov3) de la variable Pobreza están incluidos como variables de control. ${ }^{30}$

Este análisis emplea tres variables políticas para probar cinco hipótesis competitivas: las hipótesis del votante principal, de la proximidad, del cultivo,

30. La ecuación cuadrática y los términos polinomios de tercer grado captan la relación no lineal entre la variable de respuesta y las covariantes representadas por la curva de regresión no paramétrica en las figuras 2 y 3 . Una prueba de la proporción de probabilidad confirmó el efecto significativamente estadístico de estos términos para explicar el comportamiento de la respuesta. Observe que las figuras 2 y 3 representan la proporción del número de los beneficiarios de Progresa con respecto a la población municipal comparada con el nivel de pobreza para controlar la cantidad de la población. En contraste, el modelo de regresión emplea el número original de receptores por municipio para la variable dependiente con el registro de la población incluido en la parte derecha de la ecuación como control. Los coeficientes estimados en el registro de la población (tablas 2 y 3 ) están muy cerca de uno. Así, la curva prevista derivada de la regresión no divergiría perceptiblemente de la curva no paramétrica en las figuras 2 y 3. 
de recompra, y del clientelismo. Inicialmente, intento crear dos variables para probar las hipótesis del votante principal y de la proximidad. Sin embargo, resulta que estas dos variables están correlacionadas fuerte y negativamente a un nivel estadístico significativo $(r=-0.8386, p<0.05)$. Más específicamente, para la hipótesis del votante principal, elaboré la variable de la cantidad de votos del PRI para representar la fuerza del candidato, PRI, medida por la cantidad de votos del PRI en las elecciones intermedias de 1997. La variable de la proximidad es calculada sustrayendo la diferencia de votos entre los dos partidos más grandes en las elecciones intermedias de 1997 a partir de 1; una cantidad mayor significa una carrera más cerrada. ${ }^{31} \mathrm{La}$ correlación negativa entre estas variables significa que una es la inversa de la otra. Luego estimo tentativamente dos modelos por separado, uno con la variable de la cantidad de votos del PRI y la otra con la variable de la proximidad, que incluye las mismas variables del control. De hecho, los signos de los coeficientes para la cantidad de votos del PRI y la proximidad son opuestos, mientras que otras variables muestran efectos similares. Ello indica que estas dos variables son intercambiables. A fin de simplificar, he decido emplear sólo la variable de la cantidad de votos del PRI para probar las hipótesis del votante principal y la de la proximidad. Si el coeficiente es significativo con un signo positivo, ello confirma la hipótesis del votante principal. Por el contrario, si el coeficiente es significativo con un signo negativo, ello confirma la hipótesis de la proximidad.

Para probar las hipótesis del cultivo y de la recompra se emplea la variable del cambio de la cantidad de votos del PRI para representar un cambio en la cantidad de votos a favor del PRI en 1997 y en las elecciones federales de $1994 .{ }^{32}$ Esta

31. Algunos podrían argumentar que en una competencia entre tres partidos, el PRI también debería interesarse por la cantidad de votos del candidato que ocupa el tercer lugar. Por tanto, concluirían que mi medida de la proximidad, derivada solamente de los dos partidos más grandes, no fue relevante. Sin embargo, la diferencia en la cantidad de votos entre los dos partidos más grandes todavía puede captar la proximidad de una carrera entre tres o más partidos. Obsérvese que para clasificar los tipos de competencia entre partidos empleo el ENP, no el número original de partidos. Esto significa que en una competencia entre tres partidos, el PRI enfrenta a dos adversarios cuyo tamaño y fuerza relativos son casi iguales, lo que implica que estos dos adversarios obtuvieron un nivel similar de votos en la última elección. Ello significa que la diferencia de la cantidad de votos entre el primer y el segundo partido no difiere de la existente entre el primer y el tercer partido. Por tanto, la diferencia de la cantidad de votos entre el primer y el segundo candidatos puede ser una medida apropiada de proximidad, no solamente en una competencia entre dos partidos, sino también entre tres o entre varios partidos.

32. Algunos estudios emplean un cambio del número absoluto de votos en lugar de la cantidad. Sin embargo, en el periodo interelectoral la población total de votantes elegibles aumentó en 
variable se crea substrayendo la cantidad de votos del PRI en las elecciones de 1994 de la cantidad en las elecciones de 1997. Finalmente, la variable alcalde del PRI es una variable simulada para los municipios gobernados por un alcalde del PRI, codificado como 1, y para los municipios gobernados por los partidos de la oposición, codificado como 0 . Las variables y sus signos previstos aparecen resumidos en la tabla 1. En el modelo condicional, estas variables independientes interactúan con las variables simuladas para el partido hegemónico, la competencia entre dos partidos, la competencia entre tres partidos, y la competencia entre varios partidos. Si difieren los resultados en los modelos incondicionales y condicionales, ello será una fuerte evidencia de que la lógica de politización es de hecho condicional en la naturaleza de la competencia partidista.

Resumen de variables

\begin{tabular}{lcccrr}
\hline Variables & Obs. & Media & Desv. Std. & Mínimo & Máximo \\
\hline Población & 2388 & 34,342 & 99,854 & 121 & $1,633,216$ \\
Pobreza 95 & 2388 & 2.1874 & 0.9937 & 0 & 5.3458 \\
ENP & 2386 & 2.3937 & 0.5487 & 1.002 & 4.975 \\
Proximidad & 2388 & 0.7279 & 0.2087 & 0.002 & 1 \\
Cantidad de votos del PRI & 2386 & 0.5358 & 0.1467 & 0.178 & 0.999 \\
Cambio de cantidad & 2386 & -0.0649 & 0.1262 & -0.494 & 0.737 \\
de votos del PRI & & & & & \\
\hline
\end{tabular}

Tabla 1

Variables y signos previstos

\begin{tabular}{lccccc}
\hline \multicolumn{5}{c}{ Hipótesis } \\
\hline Variable & Principal & Proximidad & Cultivo & Recompra & Clientelismo \\
\hline Cantidad de votos del PRI & + & - & & & \\
Cambio de la cantidad de & & & + & - & \\
votos del PRI & & & & & + \\
Alcalde del PRI & & & & & + \\
\hline
\end{tabular}

la mayoría de los municipios. Para contar este efecto, decido emplear un cambio de la cantidad de votos. 
Resultados y discusión

En primer lugar, informo los resultados del modelo incondicional y después los resultados del modelo condicional, incorporando los efectos de configuraciones partidistas distintivas. La tabla 2 presenta los resultados de la regresión binomial negativa incondicional. Muestra que el nivel de pobreza es un determinante importante de la distribución de Progresa puesto que los coeficientes de los términos polinomios linear, cuadrático, y de tercer grado son estadísticamente significativos. Al mismo tiempo, la cantidad de votos del PRI también tiene un efecto significativo en nivel de beneficiarios de Progresa, lo cual significa que el PRI gasta más a favor de los municipios donde tienen mayor apoyo. Por otra parte, los coeficientes para el cambio de la cantidad de votos del PRI y el alcalde del PRI no son significativos. En resumen, en el modelo incondicional se apoya la hipótesis del votante principal. Estos resultados son importantes porque presentan evidencia fuerte de que Progresa beneficia a los pobres y sigue su objetivo declarado de aliviar la pobreza, pero no obstante muestra un potencial de manipulación política al estar orientado a los partidarios leales del PRI. Para explorar cómo difieren las estrategias de politización, dependiendo de las distintas presiones competitivas, el modelo condicional esclarece aún más la lógica de la politización.

Tabla 2

Resultados de la regresión binomial negativa: El modelo incondicional

\begin{tabular}{lrr}
\hline Variable & Coeficientes (S.E.) & Valores P \\
\hline Pobreza (1995) & $5.29948(0.25734)$ & $<0.000$ \\
Povsq & $-1.30359(0.11129)$ & $<0.000$ \\
Pov 3 & $0.10546(0.01473)$ & 0.000 \\
Registro (población) & $0.98236(0.02100)$ & $<0.000$ \\
Cantidad de votos del PRI & $0.49651(0.19380)$ & 0.010 \\
Cambio de la cantidad de votos del PRI & $0.06234(0.19288)$ & 0.767 \\
Alcalde del PRI (supuesto) & $-0.02124(0.05382)$ & 0.693 \\
Intercepción & $-8.08004(0.36753)$ & $<0.000$ \\
\hline
\end{tabular}

$\mathrm{N}=23832 \times$ Registro-Probabilidad $=-33921.5580 .<\mathrm{R}>$ Nota: las variables simuladas para 31 estados se incluyeron en el modelo, pero aquí no se dan a conocer los coeficientes. 
La tabla 3 divulga los resultados del modelo condicional. La primera columna muestra los resultados de la estimación de la competencia bajo la hegemonía del PRI; la segunda muestra los resultados en una competencia entre dos partidos; la tercera en una competencia entre tres partidos; y la cuarta en una competencia entre varios partidos. Lo que es consecuente con el modelo incondicional es que el nivel de pobreza es un determinante importante de la asignación de Progresa puesto que los coeficientes de los términos polinomios linear, cuadrático y de tercer grado son estadísticamente significativos, excepto en el subconjunto de competencia entre varios partidos. Sin embargo, teniendo en cuenta que el número de observaciones para la competencia entre varios partidos es muy pequeño (59 de 2,383), puede que los resultados no sean sólidos. Debe señalarse que el tamaño de los coeficientes para la variable Pobreza no varía considerablemente entre los tipos de competencia partidista. Esto significa que el nivel de pobreza es constantemente un determinante importante de los gastos de Progresa, independientemente de las configuraciones de la competencia partidista.

Por otra parte, la tabla 3 también muestra que la política importa, pero los determinantes políticos de la asignación de Progresa difieren, de acuerdo a la naturaleza de las configuraciones partidistas. Ello corrobora el debate con respecto a que las estrategias de manipulación de los recursos del PRI dependen de si enfrenta a uno, dos o más rivales serios debido a que, a medida que crece el número de partidos en competencia, es mayor el grado de incertidumbre. Teniendo en cuenta los resultados reportados en la tabla 3 , algunos de los resultados presentados en la tabla 2 parecen inexactos, como analizamos a continuación.

La prueba de la hipótesis del votante principal (y de la proximidad) muestra que la cantidad de votos del PRI tiene un efecto significativo en el nivel de beneficiarios de Progresa en una competencia entre tres partidos. Ello indica que el PRI gasta más en los municipios donde el PRI tiene mayor apoyo cuando la presión competitiva es mayor. Al comparar los coeficientes para los tipos de competencia partidista se aclara que la magnitud aumenta a medida que la carrera partidista se haga más competitiva, como se previó. Esto requiere una prueba sobre la igualdad de esos cuatro coeficientes. ${ }^{33}$ La prueba dio como resultado un rechazo de una hipótesis nula de que los coeficientes de los cuatro

33. La prueba para examinar si los coeficientes estimados para los subconjuntos de datos son iguales es la llamada prueba de Chow. 


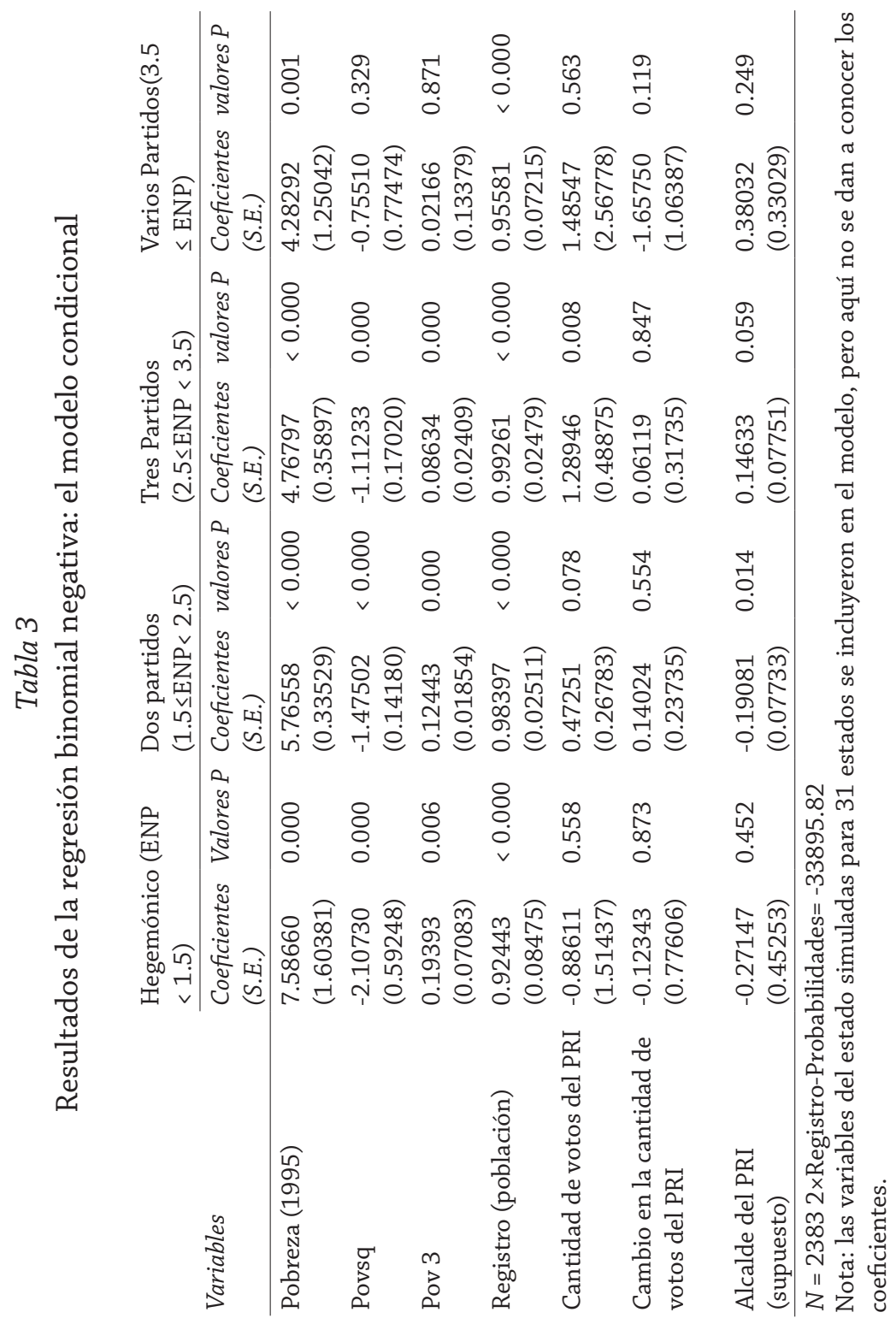


subconjuntos son iguales, confirmando que las estrategias de distribución difieren según los niveles de competencia partidista. Estos resultados apoyan la hipótesis del votante principal de que un mayor apoyo para el candidato estará asociado a mayores transferencias, y que esta predicción sería apoyada más fuertemente si el número de competidores serios aumenta debido a que el candidato tiene mayor riesgo en contra. Al mismo tiempo, significa que los resultados rechazan la hipótesis de la proximidad de que una carrera más cerrada induce mayor politización del gasto.

En contraste con un apoyo tan fuerte para la hipótesis del votante principal en los dos modelos incondicional y condicional, la prueba de la hipótesis de clientelismo identifica resultados bastante diferentes de los presentados en la tabla 2. Los resultados del modelo incondicional en la tabla 2 no muestran ningún efecto significativo de la variable del alcalde del PRI. En contraste, los resultados del modelo condicional divulgado en la tabla 3 indican que el coeficiente para el alcalde del $P R I$ es negativo a nivel estadísticamente significativo en una competencia entre dos partidos, pero positivo y casi alcanza un nivel significativo en una competencia entre tres partidos. ¿Cómo interpretamos estas muestras opuestas de los coeficientes? Es plausible que el PRI pueda tener un mayor incentivo para transferir más recursos a los municipios que son gobernados por los alcaldes del PRI cuando hace frente a un mayor grado de incertidumbre derivado de una competencia entre tres partidos. En cambio, el PRI puede tener un incentivo negativo a invertir en municipios del PRI en una competencia entre dos partidos porque, con menos presión competitiva, podría pensarse que la manipulación a nivel central era suficiente para sobrevivir a una carrera electoral. Estos resultados apoyan la hipótesis de clientelismo que predice que con un mayor grado de incertidumbre, el PRI tiene un mayor incentivo para manipular las asignaciones de gasto a través de los alcaldes del PRI que sirven como intermediarios. Para la variable de cambio de la cantidad de votos del PRI, no se observó ningún efecto significativo en ninguna de las configuraciones partidistas. Ni las hipótesis de cultivo ni la de la recompra se confirman en este análisis empírico. El PRI puede intentar manipular las asignaciones de Progresa seleccionando favorablemente los municipios que tienen un fuerte apoyo al PRI y entregando recursos más abundantes a los municipios que están gobernados por los alcaldes del PRI cuando enfrenta mayor grado de incertidumbre en una competencia entre tres partidos. 
En resumen, el análisis empírico muestra que el nivel de pobreza es ciertamente un determinante importante de los gastos para su alivio, independientemente del número de partidos en competencia. No obstante, la política también parece importar. El PRI asigna cantidades de recursos desproporcionalmente mayores a los municipios donde es mayor el apoyo político al PRI. Esta manipulación tiene más probabilidades de ocurrir en particular en una competencia entre tres partidos porque el grado de incertidumbre asociado a un resultado electoral es mayor, y de esta forma el PRI tiende a tener cada vez más riesgo en contra. Además, el PRI también intenta manipular la distribución de Progresa a través de los gobiernos municipales cuando enfrenta una competencia entre tres partidos. Este apoyo a las dos hipótesis del votante principal y del clientelismo puede implicar la posibilidad de un problema de multicolinearidad: los municipios donde es más fuerte el apoyo al PRI están gobernados por un alcalde del PRI. Sin embargo, la correlación entre estas dos variables es débil $(r=0.361)$. Ello sugiere que dos lógicas distintivas funcionan detrás de la manipulación plausible de la asignación de Progresa. Al analizar el caso de Progresa en México, este estudio muestra que la presión política proveniente de la creciente competencia electoral se ha convertido en un factor importante para explicar la politización del gasto social, incluso en la era neoliberal.

\section{Conclusión}

Este estudio intenta abordar los problemas relacionados con la distribución del gasto social, específicamente, los programas de alivio de la pobreza, en el contexto de la reestructuración política y económica. ¿Sigue teniendo importancia la política en el neoliberalismo? En caso de que así sea, ¿cuál es la lógica que impulsa la manipulación del gasto social en el modelo económico neoliberal?

Empleando el caso de un programa orientado a aliviar la pobreza en México - Progresa (1997-2000) — este estudio muestra que Progresa beneficia a los pobres en comparación con Pronasol, y que sigue su objetivo declarado de aliviar la pobreza. Ello sugiere que es probable que el neoliberalismo despolitice los programas sociales. Sin embargo, bajo la creciente competencia electoral, Progresa siguió desprestigiándose por la manipulación política que ha plagado a muchos países menos desarrollados. Además, planteo que el supuesto principal de la competencia entre dos partidos que subyace en los 
modelos teóricos de redistribución no es sostenible en muchas de las nuevas democracias, porque los sistemas partidistas aún no están consolidados, y generalmente hay más de dos partidos en competencia. El grado de incertidumbre derivado de las condiciones específicas relacionadas con las configuraciones partidistas resalta el razonamiento más preciso del incentivo de un partido a manipular las asignaciones del gasto. Un modelo econométrico que incorpore el efecto de tales configuraciones partidistas muestra que el PRI puede responder en una competencia entre tres partidos, en la cual la incertidumbre es mayor, asignando más favorablemente los beneficios a los municipios donde se concentra el apoyo político al PRI y gastando de forma más favorable en dichos municipios del PRI. Al demostrar estos resultados, este análisis proporciona evidencia de que la politización del gasto social parece ocurrir continuamente en la era neoliberal cuando hay mayor presión electoral.

Sin embargo, se requieren otros análisis para explicar completamente la lógica que existe detrás de la politización potencial de los programas sociales. Primero, este estudio encontró evidencias para mostrar que el PRI presta atención al partidismo de los municipios a la hora de decidir dónde gastar. Sin embargo, no explica en qué forma los alcaldes intervienen en el proceso (a) de recibir transferencias del gobierno federal y (b) entregar los recursos a los receptores dentro de los municipios. Sólo sugiere la posibilidad de que los beneficios de Progresa sean distribuidos a través de las redes patróncliente por los alcaldes del PRI. Además, sigue existiendo una posibilidad de que el alcalde intervenga independientemente de la toma de decisiones a nivel central. Es decir, como planteamos anteriormente, en el proceso de selección del beneficiario, primero se recopila la información sobre los beneficiarios elegibles a nivel de localidad y luego se envía a la oficina central de Progresa. Así, el efecto significativo del partidismo de los municipios en el análisis empírico puede ser explicado por esta manipulación a un nivel local, no por la manipulación a nivel central. La posibilidad de que la manipulación a nivel local ocurra independientemente de la intención del gobierno central se refuerza por el hecho de que en el periodo de Progresa, la autoridad presidencial del PRI estaba debilitada e incluso puede que los alcaldes del PRI hayan actuado de forma autónoma. Para investigar esta posibilidad, este análisis cuantitativo debe complementarse con un estudio de caso cualitativo para examinar la motivación y el comportamiento de los actores municipales. Ello proporcionaría una fuerte evidencia de que el neoliberalismo promueve la elaboración de 
políticas tecnócratas a nivel del gobierno central, mientras las prácticas políticas tradicionales persisten a nivel local (Fox, 1994; Snyder, 2001).

Este estudio extrae inferencias de un grupo de datos representativos de un año de observaciones. Para generalizar los resultados y confirmar la hipótesis de manera más rigurosa, sería beneficioso incorporar variaciones con el paso del tiempo empleando además datos anuales. Una comparación a lo largo del tiempo es particularmente importante debido a que, a pesar de que este estudio muestra que sigue habiendo manipulación, debe señalarse que la frecuencia y el grado de gasto politizado han disminuido significativamente en comparación con el programa anterior contra la pobreza, Pronasol. Además, durante la administración de Fox, Progresa fue renombrado como Oportunidades en el año 2002, lo que parece haberlo despolitizado aún más ${ }^{34}$ Una comparación de cómo se distribuyen Pronasol, Progresa y Oportunidades ayudaría a entender lo que explica la lógica de despolitización plausible a lo largo del tiempo. ${ }^{35}$

Finalmente, este análisis aclara exclusivamente en el caso de Progresa en México la lógica de la politización neoliberal del gasto social. En años recientes, otros países de América Latina y el Caribe han introducido programas para orientar los programas condicionales de transferencia de efectivo en un contexto similar al de México, es decir, el proceso simultáneo de democratización política y liberalización económica: el programa Bolsa Escola en Brasil, el programa Familias en Acción en Colombia, el programa Asignación Familiar en Honduras, el programa Red de Protección en Nicaragua, el Programa de Avance a través de Salud y la Educación en Jamaica, y muchos otros (Rawlings, 2004). Un análisis de los procesos políticos detrás de la introducción de esos programas y una comparación de los resultados

34. Antes de las elecciones federales de 2006, una organización independiente realizó una investigación a gran escala sobre el empleo político de programas gubernamentales, en colaboración con Sedesol. Informó que a escala local, los políticos y/o sus aliados aún manipulan el proceso de selección del beneficiario y/o la asignación con fines electorales en algunas regiones, pero Oportunidades es menos favorable a estas maniobras políticas que otros programas contra la pobreza (Fundar, 2006). Esto podría ser explicado por el esfuerzo explícito de Sedesol para promover una campaña a escala nacional de que los beneficios de Oportunidades no están condicionados por la opción de voto de una persona (Sedesol, 2001 y 2005).

35. Uno de los factores plausibles es el compromiso activo de los actores civiles de la sociedad en la supervisión del uso político de los programas sociales para las elecciones. Debe señalarse que la actividad de Alianza Cívica, una ONG mexicana, ha sido organizar las observaciones electorales desde 1994. Dicha ONG ha difundido información sobre irregularidades electorales y votantes adiestrados para no consentir la compra del voto. 
proporcionaría una explicación más sistemática de la lógica política que subyace en la orientación de las políticas sociales que cada vez se hace más popular en la región.

Como es bien conocido, proporcionar evidencia sólida de prácticas políticas tales como la compra de voto, la corrupción y la manipulación del gasto social es empíricamente difícil. Lo que pueden hacer los investigadores es no confirmar sino sólo aproximarse tanto como sea posible a la causalidad plausible. Para obtener mayor plausibilidad, el estudio debe incorporar la agenda de la investigación anterior en análisis futuros. A pesar de estas limitaciones, al emplear un análisis altamente desagregado y un modelo econométrico cuidadosamente elaborado, este estudio es uno de los pocos que intenta esclarecer la lógica que impulsa la politización plausible del gasto social en la era del neoliberalismo. my

\section{Referencias bibliográficas}

Adato, Michelle, David Coady y Marie Ruel, Final Report: An Operations Evaluation of Progresa from the Perspective of Beneficiaries, Promoters, School Directors, and Health Staff, International Food Policy Research Institute, Washington, DC, 2000.

Alianza Cívica, Informe preliminar de Alianza Cívica sobre la calidad de la jornada electoral del 2 de julio de 2000, Alianza Cívica, Ciudad de México, 2000.

Bruhn, Kathleen, “Social Spending and Political Support: The 'Lessons' of the National Solidarity Program in Mexico", Comparative Politics, vol. 28, núm. 2, 1996, pp. 151-77.

BRusco, Valeria, Marcelo Nazareno y Susan C. Stokes, "Vote Buying in Argentina”, Latin American Research Review, vol. 39, núm. 2, 2004, pp. 66-88.

CALvo, Ernesto y María Victoria Murillo, "Who Delivers? Partisan Clients in the Argentine Electoral Market", American Journal of Political Science, vol. 48, núm. 4, 2004, pp. 742-757.

Cameron, A. Colin y Pravin K. Trivedi, "Econometric Models Based on Count Data: Comparisons and Applications of Some Estimators and Tests", Journal of Applied Econometrics, vol. 1, núm. 1, 1986, pp. 29-53.

- - Regression Analysis of Count Data, Cambridge University Press, Nueva York, 1998. 
CASE, Anne, "Election Goals and Income Redistribution: Recent Evidence from Albania”, European Economic Review, vol. 45, núm. 3, 2001, pp. 405-23.

Consejo Nacional de Población (Conapo), Índices de Marginación, Conapo, Ciudad de México, 1995.

Cox, Gary W. y Mathew D. McCubbins, "Electoral Politics as a Redistributive Game”, Journal of Politics, vol. 48, núm. 2, 1986, pp. 370-389.

DiXIT, Avinash y John Londregan, "The Determinants of Success of Special Interests in Redistributive Politics", Journal of Politics, vol. 58, núm. 4, 1996, pp. 1132-55.

Dresser, Denise, Neopopulist Solutions to Neoliberal Problems: Mexico's National Solidarity Program, Center for Us-Mexican Studies, University of California San Diego, San Diego, 1991.

Escobar Latapí, Agustín, "The Progresa Programme and Social Change in Rural Mexico", Social Policy Reform and Market Governance in Latin America, Louise Haagh y Camilla T. Helø (eds.), Palgrave Macmillan, Basingstoke y Nueva York, 2002, pp. 219-241.

Fox, Jonathan, "The Difficult Transition from Clientelism to Citizenship", World Politics, vol. 46, núm. 2, 1994, pp. 151-84.

Fundar. Centro de Análisis e Investigación, Monitoreo de programas sociales en contextos electorales, Fundar, Ciudad de México, 2006.

GedDes, Barbara, Politician's Dilemma: Building State Capacity in Latin America, University of California Press, Berkeley y Los Ángeles, 1994.

Graham, Carol, "The Politics of Protecting the Poor during Adjustment: Bolivia's Emergency Social Fund”, World Development, vol. 20, núm. 9, 1992, pp. 1233-51.

HANUSHEK, Eric A. y John E. Jackson, Statistical Methods for Social Scientists, Academic Press, Nueva York, 1977.

HerediA, Blanca, "Clientelism in Flux: Democratization and Interest Intermediation in Contemporary Mexico". Ponencia presentada en The $20^{\text {th }}$ International Conference of the Latin American Studies Association, 17-19 de abril, en Guadalajara, Mexico, 1997.

Hiskey, Jonathan T., "Demand-Based Development and Local Electoral Environment in Mexico”, Comparative Politics, vol. 36, núm. 1, 2003, pp. 41-59.

Inter-American Development Bank, "The Story behind Oportunidades", IDBAmerica (octubre), 2004. 
LAAKso, Markku y Rein Taagepera, “'Effective' Number of Parties: A Measure with Application to West Europe", Comparative Political Studies, vol. 12, núm. 1, 1979, pp. 3-27.

Laurell, Asa Cristina, "The Transformation of Social Policy in Mexico", Kevin J. Middlebrook y Eduardo Zepeda (eds.), Confronting Development: Assessing Mexico's Economic and Social Policy Challenges, Stanford University Press, Stanford, 2003, pp. 320-349.

LEVy, Santiago, "Poverty Alleviation in Mexico", World Bank Working Paper Series, núm. 679, World Bank, Washington, DC, 1991.

- - Progress against Poverty: Sustaining Mexico's PROGRESA-OPORTUNIDADES Program, Brookings Institution Press, Washington, DC, 2006.

LiNDBECK, Assar y Jörgen Weibull, "Balanced-Budget Redistribution as the Outcome of Political Competition”, Public Choice, núm. 52, 1987, pp. 272-97.

Magaloni, Beatriz, Alberto Díaz-Cayeros y Federico Estévez, "The Erosion of Party Hegemony, Clientelism and Portfolio Diversification: The Program National Solidarity (Pronasol) in Mexico". Documento de trabajo, 2003. Disponible en: http://www.stanford.edu/ albertod/cientelismEstevezMagaloniDiazApril16.pdf. Fecha de consulta: 15 de diciembre de 2004.

MARISCAL, Ángeles, "En Chiapas, documentan desvío del erario en favor de Labastida", La Jornada, 30 de mayo, 2000.

McCullagh, Peter y John A. Nelder, Generalized Linear Models, Chapman and Hall, Nueva York, 1989.

Mebane, Walter R. Jr. y Jasjeet S. Sekhon, "Robust Estimation and Outlier Detection for Overdispersed Multinomial Models of Count Data", American Journal of Political Science, vol. 48, núm. 2, 2004, pp. 392-411.

MedinA, Luis Fernando y Susan C. Stokes, "Clientelism as Political Monopoly". Ponencia presentada en el año 2000 en el Annual Meeting of the American Political Science Association, Boston, 29 de agosto $a l 1^{\circ}$ de septiembre, 2002.

MoLINAR, Juan y Jeffrey Weldon, "Electoral Determinants and Consequences of Nation Solidarity", Wayne Cornelius, Ann Craig y Jonathan Fox (eds.), Transforming State-Society Relations in Mexico: The National Solidarity Strategy, Center for Us-Mexican Studies, UCSD, La Jolla, 1994, pp. 123-141.

PARDINAS, Juan, "Fighting Poverty in Mexico: Policy Challenges", Luis Rubio y Susan Purcell Kaufman (eds.), Mexico under Fox, Lynne Reinner Publishers, Boulder, 2004, pp. 65-86. 
Poder Ejecutivo Federal, Progresa: Programa de Educación, Salud, y Nutrición. Documento para uso interno, s/f.

Pozo, Blanca del y Ricardo Aparicio, Estudios sobre la participación ciudadana $y$ las condiciones del voto libre y secreto en las elecciones federales del año 2000: una aproximación a la magnitud de la inducción y coacción del voto, Mimeo, 2001.

R Development Core Team, R: A Language and Environment for Statistical Computing (versión 1.9.1.), R Foundation for Statistical Computing, Vienna, Austria, 2004.

RAWLings, Laura, "A New Approach to Social Assistance: Latin America's Experience with Conditional Cash Transfer Programs", Social Protection Discussion Paper Series núm. 416, World Bank, Washington, DC, 2004.

Roberts, Kenneth M., "Neoliberalism and the Transformation of Populism in Latin America: The Peruvian Case", World Politics, núm. 48, octubre, 1995, pp. 82-116.

_- , "Social Inequalities without Class Cleavages in Latin America's Neoliberal Era", Studies in Comparative International Development, vol. 36, núm. 4, 2002, pp. 3-33.

RobInson, James A. y Thierry Verdier, "The Political Economy of Clientelism”, Working Paper 3205, Public Policy, Center for Economic Policy Research, 2003.

RochA Menocal, Alina, "Do Old Habits Die Hard? A Statistical Exploration of the Politization of Progresa, Mexico's Latest Federal Poverty-Alleviation Programme, under the Zedillo Administration", Journal of Latin American Studies, núm. 33, 2001, pp. 513-38.

RuEdA, David y Jonas Pontusson, "Wage Inequality and Varieties of Capitalism”, World Politics, vol. 52, núm. 3, 2000, pp. 350-83.

ScotT, John, Progresa: contexto y relevancia. Documentos de trabajo. CIDE, Ciudad de México, 2003.

SCHADY, Norbert R., "The Political Economy of Expenditures by the Peruvian Social Fund (Foncodes), 1991-95”, American Political Science Review, vol. 94, núm. 2, 2000, pp. 289-304.

Secretaría de Desarrollo Social, Guía para la candidata a beneficiaria: lo que necesita saber del Programa de Educación, Salud, y Nutrición, Secretaría de Desarrollo Social, Ciudad de México, 2001.

_- El Elindaje electoral del programa Oportunidades, Secretaría de Desarrollo Social, Ciudad México, 2005. 
Shadlen, Kenneth C., "Neoliberalism, Corporatism, and Small Business Political Activism in Contemporary Mexico", Latin American Research Review, vol. 35, núm. 2, 2000, pp. 73-106.

SkoufIAS, Emmanuel, Benjamin Davis y Sergio de la Vega, "Targeting the Poor in Mexico: An Evaluation of the Selection of Households into PROGRESA", World Development, vol. 29, núm. 10, 2001, pp. 1769-84.

SNYDER, Richard, Politics after Neoliberalism: Reregulation in Mexico, Cambridge University Press, Cambridge, 2001.

Stokes, Susan C., "Perverse Accountability: A Formal Model of Machine Politics with Evidence from Argentina”, American Political Science Review, vol. 99, núm. 3, 2005, pp. 315-325.

TreJo, Guillermo y Claudio Jones, "Political Dilemmas of Welfare Reform: Poverty and Inequality in Mexico", Susan Purcell Kaufman y Luis Rubio (eds.), Mexico under Zedillo, Lynne Rienner Publishers, Boulder, 1998, pp. 67-97.

Valenzuela, Arturo, Political Brokers in Chile: Local Government in a Centralized Polity, Duke University Press, Durham, 1977.

WeYLAND, Kurt, "Neopopulism and Neoliberalism in Latin America: Unexpected Affinities", Studies in Comparative International Development, vol. 33, núm. 3, 1996, pp. 3-31.

Zedillo Ponce de León, Ernesto, Sexto Informe de Gobierno, Presidencia de la República, Ciudad de México, 2000. 